\title{
Characterization of impact behaviour of armour plate materials
}

\author{
M.N. Bassim ${ }^{1}$, M. Bolduc ${ }^{2}$, G. Nazimuddin ${ }^{1}$, J. Delorme ${ }^{1}$, and I. Polyzois ${ }^{1}$ \\ 1 University of Manitoba, Winnipeg, Canada, R3T 5V6 \\ ${ }^{2}$ Department of National Defense of Canada, DRDC Valcartier, Quebec, Canada
}

\begin{abstract}
Three armour plate materials, including two steels, namely HHA and Mars 300, and an aluminium alloy 5083, were studied under impact loading to determine their behaviour and the mechanisms of deformation that lead to failure. The experimental testing was carried out using either a direct impact compression Split Hopkinson Bar or a torsion Hopkinson Bar. The impact properties and stress-strain cures were obtained as a function of the impact momentum in compression and the angle of twist in torsion. It was found that at the high strain rates developed in the specimen during the tests, the deformation occurs by the formation of adiabatic shear bands (ASBs) which may lead to the formation of cracks within the bands and the ultimate failure of the specimens. It was also found that below a certain impact momentum, the deformation is more uniform and no ASBs are formed. Also, ASBs are more likely to form in the BCC metals such as the two steels while diffuse ASBs associated with plastic flow are exhibited in the 5083 aluminum alloy. Microstructural techniques ranging from optical microscopy to atomic force microscopy (AFM) were used to study the topography of the ASBs. Also, modelling of the formation was performed. The results provide a comprehensive understanding of the role of ASBs in the failure of these materials.
\end{abstract}

\section{Introduction}

Candidate materials with potential use as armour plates have to withstand the impact of high speed projectiles and absorb the shock produced by such impacts. Materials to be used for this purpose have ranged from high strength steels, aluminum alloys, as well as metal matrix composites and modern ceramics. Such materials have to withstand high strain rates and large strains of values up to two. Testing at these strain rates in excess of 103 s-1 and large strains is usually carried out using Split Hopkinson Bars in different configurations such as direct impact compression bar and torsional bars.

An important microstructural damage feature in materials exposed to such strains and strain rates is the occurrence of adiabatic shear bands (ASBs). They are regions where most of the shear strains are localized. They are observed in many applications involving machine chips [1], friction welding, explosive welding, rail surface wear [2] and in ballistic impact and fracture as well as dynamic deformation [3,4].

Adiabatic shear bands are manifestations of nonequilibrium thermodynamic events. At large strain and high strain rates, metallic materials exhibit, to various degrees, depending on the mechanical and thermodynamic properties, a combination of strain hardening and, at one point, thermal softening which is caused by adiabatic healing which causes significant rise in temperature in the material. At times, this rise may cause phase transformations, and in steels, the formation of martensitic structures.

Adiabatic shear bands are also the precursor of crack initiation which may lead to the fracture of the specimen or structure. While in many situations, ASBs produce strings of brittle material in the structure, the rise in temperature may lead to observation of ductile fracture characterized by the presence of dimples [5].

The experimental likelihood of occurrence of ASBs is governed by several factors. Initial heat treatment of the material may produce different microstructures and corresponding mechanical properties. The ASB occurrence increases with the yield strength and hardness. For more ductile materials, the strain rates required for formation of ASBs is higher than for harder materials. Also, the formation of ASBs is controlled by the material temperature and impact momentum. Thus, at high enough temperatures and low impact momentum, ASBs may not form at all.

The present study details evaluation of the mechanisms of deformation and failure of candidate armour materials of interest to the Department of National Defense of Canada. These metallic materials range from high strength steels to an aluminum alloy. Beside an extensive experimental study, modeling and simulation to determine the rise in temperature in the specimen, was undertaken using two approaches, namely the Johnson-Cook model, and a model developed by the author of this current study which predicts the occurrence of ASBs and the temperature rise associated with it.

\section{Experimental procedure}

The research was carried out to perform an evaluation of three armour materials using both a direct compression Hopkinson Bar and a torsional Hopkinson Bar. The three materials are maraging 300 steel, HHA steel and 5083 aluminum alloy. The impact resistance was evaluated as a function of impact momentum. This was followed by microstructural examination of the impacted specimens to determine the mechanisms of failure as a function of evolution of adiabatic shear bands. Modeling of the process of formation of adiabatic shear bands and the rise in temperature was next studied. Two approaches were used, a thermoplastic resistance model [6] based on Anand [7], and application of the Johnson-Cook model [8]. 


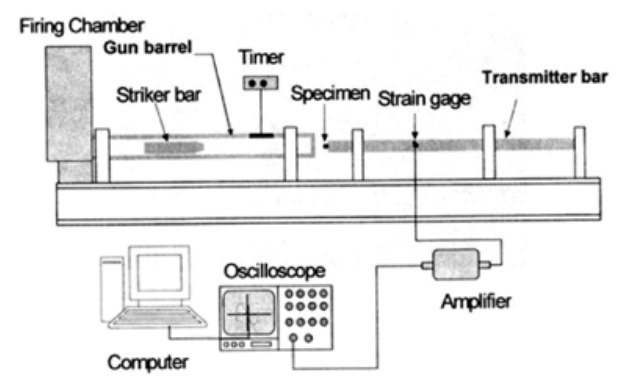

Fig. 1. Direct impact Split Hopkinson Bar.

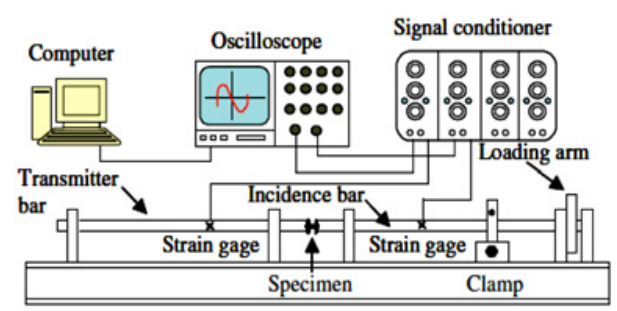

Fig. 2. Torsional Hopkinson Bar.

\subsection{Experimental impact testing}

The tests for evaluation of the impact resistance of the armour materials were carried out either using a direct impact compression Hopkinson Bar, shown in figure 1 or a torsional Hopkinson Bar shown in figure 2. For the compression tests, the firing pressure was varied so that the projectile could strike the samples at impact velocities and thus impact momenta, ranging from 20 to $30 \mathrm{~m} / \mathrm{s}$. Stressstrain curves of the impact were reconstructed from the strain-time signals obtained from the transmitter bar.

The torsional bar employs stored-torque technique of loading in which the loading torque is stored between the clamp and the loading arm using various angles of twist. The clamp is sufficiently tightened prior to loading to prevent rotation of the incident bar as pure torsion load is applied by a hydraulic jack connected to a rotating wheel attached to the loading arm. On reaching the desired angle of twist, the clamp is further tightened until the load release pin and the stored torque is released and generates elastic waves, which travel along the incident bar and deform the specimen. The strain rate, shear strain and shear stress were calculated using the following equations,

$$
\begin{gathered}
\dot{\gamma}_{s}(t)=\frac{2 C D_{x}}{L_{s} D} \gamma_{R}(t) \\
\gamma_{s}(t)=\frac{C D_{x}}{L_{s} D} \sum\left[\gamma_{T}(t)-\left\{\gamma_{I}(t)-\gamma_{R}(t)\right\}\right] \Delta T \\
\tau_{s}(t)=\frac{G D^{3}}{8 D_{s}^{2} \tau_{s}} \gamma_{T}
\end{gathered}
$$

where $\dot{\gamma}_{s}(t)$ is the shear strain rate, $\gamma_{s}(t)$ is the shear strain, $\tau_{s}(t)$, is the shear stress, $D_{s}$ is the mean diameter of the thin walled specimen and $L_{s}$ is its length, $D$ is the diameter of the incident and transmitter bar, and $C$ is the velocity of the wave propagation in the bar. Variables $\gamma_{T}(t), \gamma_{I}(t)$, and $\gamma_{R}(t)$ are the shear strains measured, where $\gamma_{T}(t)$ is the transmitted shear, $\gamma_{I}(t)$ is the incident and $\gamma_{R}(t)$ is the reflected shear strain.

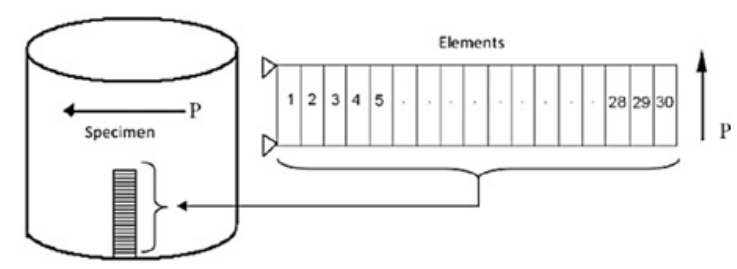

Fig. 3. Finite element mesh for torsional specimen.

\subsection{Microstructural examination}

All impacted specimens were prepared for microstructural examination using a Zeiss optical microscope. Specimens from impact experiments were thoroughly examined for defects in the microstructure such as micro-cracks, shear bands and cracks. Valuable information such as deformation and reorientation of the grains as well as presence of adiabatic shear bands were recorded and documented.

The specimens were also examined using scanning electron (SEM) and atomic force microscopy (AFM). The technique involves using a tip (stylus) mounted on one end of a cylindrical peizo-electric tube. The tube contains X, Y and $\mathrm{Z}$ electrodes. A motor attached to a lead screw moves the specimen relative to the stylus and maps up the surface of the specimen.

\subsection{Modelling and simulation}

Two models have been used in this investigation. One developed by Feng and Bassim [6] uses a rate dependent equation developed by Anand [7]. The main equations are found in [6] and will not be repeated here. The mesh for a torsional specimen is shown in figure 3 .

The other model is the Johnson-Cook model. This model is dependent on experimental data since it is an empirical and phenomenological model. In this model, hardening is isotropic and the dynamic flow stress $\sigma_{0}$ is given as

$$
\sigma_{0}=\left[A+B\left(\bar{\varepsilon}^{p i}\right)^{n}\right]\left[1+C \ln \left(\frac{\bar{\varepsilon}^{\dot{p} i}}{\varepsilon_{0}}\right)\right]\left[1-\hat{T}^{m}\right]
$$

where $\bar{\varepsilon}^{p i}$ is the equivalent plastic strain, $A$ is the static yield strength, $B$ is the strain hardening coefficient, $n$ is the strain hardening exponent, $\mathrm{m}$ is the thermal sensitivity parameters and $\hat{T}$ is the non-dimensional homologous temperature defined as $\hat{T}=\left(\frac{T-T_{R E F}}{T_{m}-T_{R E F}}\right)$ and $T_{m}$ is the melting temperature.

\section{Results and discussion}

\subsection{Stress-strain curves}

The stress-strain curves for all testing performed on the three armour materials were obtained. Typical impact data from testing of the steel armour plates, namely maraging 300 steel and HHA steel are shown in figures 4 and 5, while figure 6 shows the stress-strain curve of 5083 aluminum alloy. 


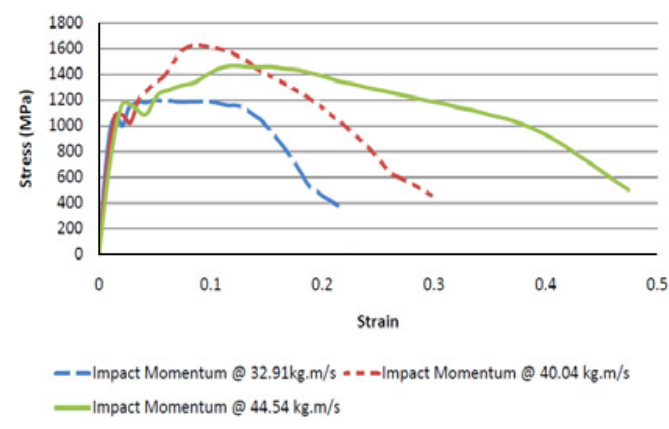

Fig. 4. Stress-strain curve for maraging steel 300 in compression.

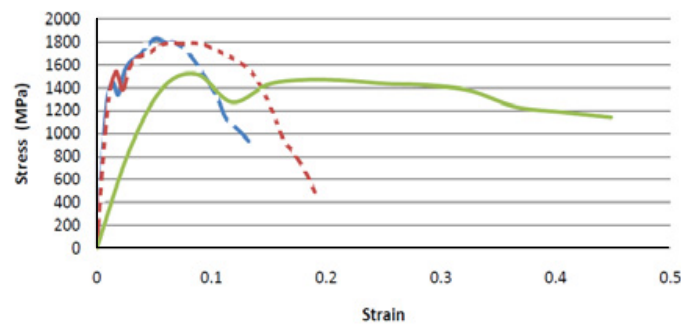

- -Impact Momentum @ $32.91 \mathrm{~kg} \cdot \mathrm{m} / \mathrm{s} \cdots \cdots$... Impact Momentum @ $40.24 \mathrm{~kg} \cdot \mathrm{m} / \mathrm{s}$ —Impact Momentum @ $45.49 \mathrm{~kg} \cdot \mathrm{m} / \mathrm{s}$

Fig. 5. Stress-strain curve for HHA steel in compression.

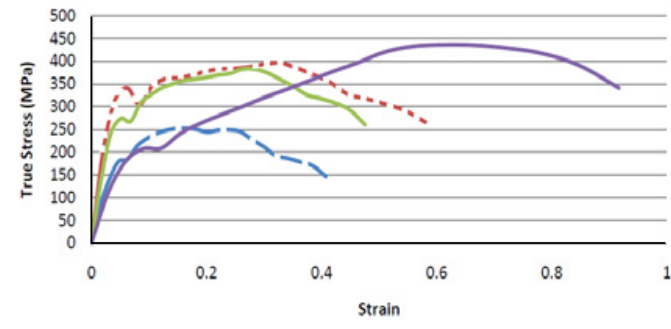

- -Impact Momentum @ $20.34 \mathrm{~kg} \cdot \mathrm{m} / \mathrm{s}=\cdots \cdot \mathrm{m}$ mact Momentum @ $28.63 \mathrm{~kg} \cdot \mathrm{m} / \mathrm{s}$

—Impact Momentum @ $32.91 \mathrm{~kg} \cdot \mathrm{m} / \mathrm{s}$ — Impact Momentum @ $40.19 \mathrm{~kg} \cdot \mathrm{m} / \mathrm{s}$

Fig. 6. Stress-strain curve for 5083 aluminum alloy in compression.

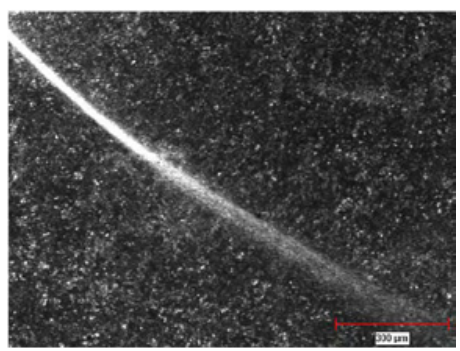

Fig. 7. Adiabatic shear band in maraging steel 300 .

\subsection{Microstructural features}

Figure 7 shows a well-defined ASB from maraging steel while figure 8 shows diffuse ASB in the aluminum alloy. Figure 9 shows an atomic force microscopic image of an ASB in maraging 300 steel. The uneven surface roughness shows a more densely packed mass in the region of the shear band than in the adjacent regions.

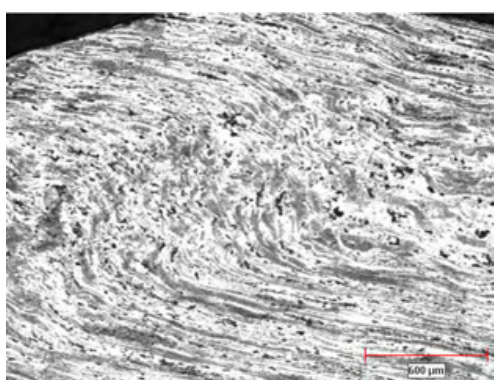

Fig. 8. Adiabatic shear band in 5083 aluminum.

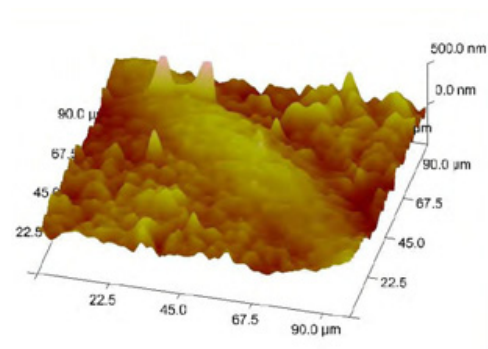

Fig. 9. Atomic force microscope image of adiabatic shear band in maraging steel 300 .

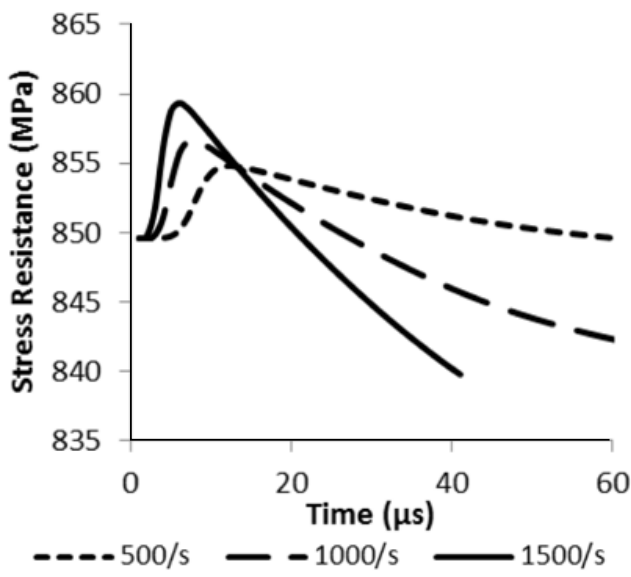

Fig. 10. Stress-resistance curve for maraging steel 300.

\subsection{Modelling and simulation}

A typical stress-resistance curve for maraging 300 steel obtained from the finite element analysis of a torsion specimen is shown in figure 10 while the temperature rise in the specimen for three strain rates is given in figure 11. Figure 10 shows an initial rise in the stress resistance corresponding to work hardening followed by a decrease in stress resistance as thermal softening becomes predominant. This delineates the onset of formation of adiabatic shear bands in the steel.

The use of the Johnson-cook model to predict the onset of formation of ASBs is shown in figure 12. It is clear that the Johnson-cook model, as used, does not show the effect of thermal softening. It is worth noting that the maximum shear criterion can be used to determine the time at which shear failure occurs. 


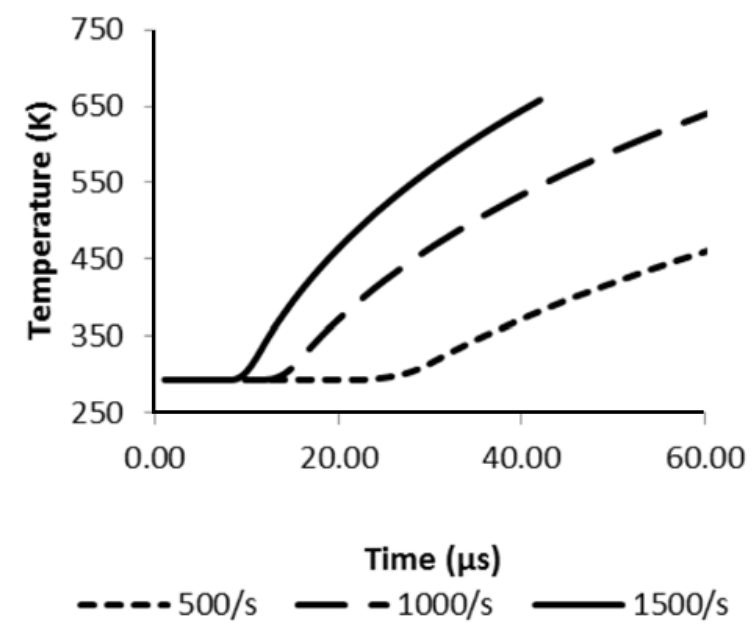

Fig. 11. Temperature rise in the specimen.

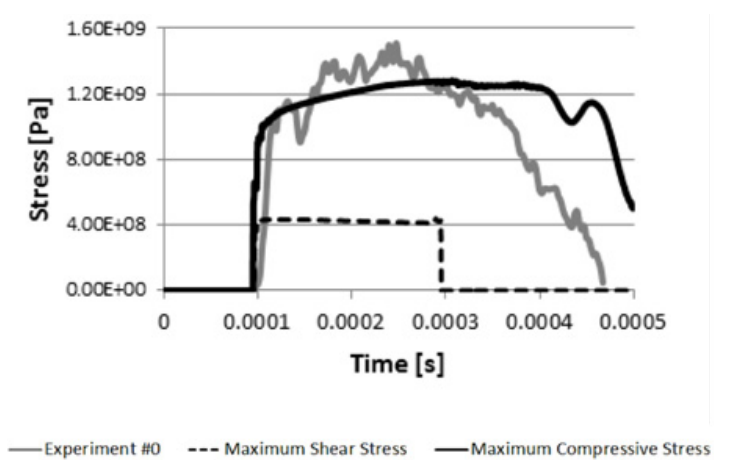

Fig. 12. Johnson-Cook result and maximum shear model for stress-strain curve of maraging steel 300.

The results show that, from a microstructural viewpoint, body-centered cubic metals (steel, such as maraging 300 steel and HHA) exhibit well formed ASBs while FCC metals, which are generally more ductile, shows only diffuse ASBs due to plastic flow occurring during strainhardening. This observation was made also in copper by Yazdani [9].

\section{Conclusions}

This study provides a comprehensive characterization of the impact behavior of three armour materials, namely maraging 300 steel, HHA steel, and 5083 aluminum alloy. Stress-strain curves in compression and torsion were obtained. Microstructural examination revealed the occurrence of adiabatic shear bands which were more defined in the steel and were diffuse in the aluminum alloy. A plastic model developed by Feng and Bassim predicts the occurrence of adiabatic shear bands while the Johnson-Cook model predicts the work hardening part of the stress-strain curve. A maximum shear model predicts more accurately the occurrence of adiabatic shear bands.

\section{Acknowledgements}

This study was sponsored and funded by the Department of National Defense of Canada.

\section{References}

1. J. T. Black and R. A. Kohser, "DeGarmo's Materials and Processes in Manufacturing", E 10, Wiley, (2007)

2. R. Nakkalil, J. R. Hornaday and M. N. Bassim, Mat. Sci. Eng. A, 141 (1991) 247-260

3. X. W. Chen, Q.M. Li and S.C. Fan, Int. J. Impact Eng. 31 (2005) 877-893

4. M.N. Bassim, J. Mat. Proc. Tech, 119 (2001) 234-236

5. A.G. Odeshi, M.N. Bassim, Mat. Sci. Eng A, 525 (2009) 96-101

6. H. Feng and M.N. Bassim, Mat. Sci. Eng A, 266 (1999) 255-260

7. L. Anand, Int. J. Plasticity, 1 (1985) 213-231

8. J.R. Johnson and W.H. Cook, Eng. Fract. Mech., 21 (1985) 31-48

9. F. Yazdani, M.N. Bassim and A.G. Odeshi, Procedia Eng., 1 (2009) 225-228 